wonnenen Normalserumprotein. Zwischen Harnund Serumalbumin bestehen keine erheblichen Differenzen. Das Aibumin der Lipoidnephrose ist ein besonders leicht spaltbares Protein, wie der folgende Versuch mit $p$-Toluolsulfonchlorid-Natrium zeigt, das bekanntlich in wäßriger Lösung Hypochlorit entwickelt. Diese Hypochloritmengen sind offenbar sehr gering, denn bei der Reaktion mit dem gleichen Eiweißanteil von Gesunden und auch von Patienten mit chron. Nephritis läßt sich keine $\mathrm{CO}_{2}$ - oder $\mathrm{N}_{2}$-Entwicklung nachweisen.

Wie sich der Abbau der Proteine im einzelnen vollzieht, muß noch näher untersucht werden.
Doch hat schon $\mathrm{Dak}$ i $\mathbf{n}^{6}$ darauf hingewiesen, daß bei der Einwirkung von Chloramin $T$ auf Aminosäuren intermediär verschiedene Reaktionsprodukte entstehen können. Die vorliegenden Ergebnisse sprechen dafür, daß bei manchen Erkrankungen Proteine vorkommen, die gegenüber Hypochlorit resistenter sind als normale und solche, die wesentlich leichter zerstört werden. Die Ursachen für dieses verschiedene Verhalten sind noch nicht klar. Wahrscheinlich können sie in strukturellen Veränderungen der Proteine gesucht werden.

${ }^{6}$ H. D. D a k in, Biochemic. J. 11, 79 [1917].

\title{
Tri- und Tetrazoliumsalze als Reduktionsindikatoren bei Meerestieren in verschiedenen Entwicklungsstadien
}

\author{
Von Hans-Joachim Bielig und Hans Querner \\ Aus dem Kaiser-Wilhelm-Institut für medizinische Forschung Heidelberg, Institut für \\ Chemie, und dem Zoologischen Institut der Universität Heidelberg \\ (Z. Naturforschg. 4 b, 21-23 [1949]; eingegangen am 7. März 1949)
}

$\mathrm{N}$ ach $\mathrm{Kuhn}$ und Jer che ${ }^{1}$ reduzieren Bakterien, Hefen, keimende Samen, Keimpflanzen und die Wurzelspitzen von Kressepflanzen farblose Tetrazoliumsalze vom Typ I-III zu roten Formazanen. Die elektive Rotfärbung durch Triphenylformazan (I) bei Embryonen verschiedener Pflanzensamen bildet nach Lakon" die Grundlage eines von der „Keimreife“ des Samens unabhängigen Keimfähigkeitsnachweises. Rötung mit 5-Furfuryl-2.3-diphenyl-tetrazoliumchlorid geben u. a. auch der Liquor des Stierspermas sowie Chalazen und Blastoderm des Hühnereies ${ }^{3}$. Der Test wurde ferner auf normales und neoplastisches menschliches Gewebe angewandt ${ }^{4}$. In Coli- und Typhusbakterien findet man das Formazan an spezifischen „Reduktionsorten“, die Gebiete hoher Nucleinsäure-Konzentration (Nucleoide) einschließen und der Einwirkung von Bakteriophagen Widerstand leisten ${ }^{5}$.

1 R. Kuhn u. D. Je r chel, Ber. dtsch. chem. Ges. 74, 949 [1941]. - D. J e r c h e l, Naturforschg. u. Medizin in Deutschland 1939-1946, Bd. 39, S. 59, Wiesbaden 1947.

2 G. L a k o n, Ber. dtsch. bot. Ges. 60, 299, 343 [1942]. - R. H. Porter, M. Durrellu. H. J. R o m m, Plant Physiol. 22, 149 [1947]. - H. J. Cot tr e l l, Nature [London] 159, 748 [1947]. - T. D. W a u g h, Science [New York] 108, 275 [1948].

3 A. M. M a t t son, C. O. Jensen u. R. A. D u t c h e r, Science [New York] 106, 294 [1947].
Unsere Untersuchungen an der Biologischen Anstalt Helgoland (z. Zt. List/Sylt) haben jetzt ergeben, daß sich Tri- und Tetrazoliumsalze auch bei Tieren in verschiedenen Entwicklungsstadien als Reduktionsindikatoren bewähren. Die Triazoliumsalze (vgl. VI) wurden von $\mathrm{Ludolphy^{6 }}$ dargestellt. Sie werden von keimfähigen Pflanzensamen zu roten Amino-azokörpern der Naphthalinreihe reduziert. Geprüft wurde die Einwirkung der folgenden Substanzen auf einige Arten von Echinodermen, Mollusken, Anneliden, Coelenteraten, Crustaceen und Protozoen: 2.3.5-Triphenyltetrazoliumchlorid(I), 5-Methyl-2.3-diphenyl-tetrazoliumchlorid (II), 5 - Athyl-2.3 - diphenyl-tetrazoliumchlorid (III), 5.5' - Dimethyl - 3.3' - diphenyl$2.2^{\prime}$ - di - $p$-diphenylen - ditetrazoliumchlorid (IV) ${ }^{6}$, $2.2^{\prime}$ - Diphenyl - 3.3' - bis - (4 - carbaethoxy - phenyl) $5.5^{\prime}$ - $p$ - phenyl-ditetrazoliumchlorid $(\mathrm{V})^{7}$ und 1 Phenyl-2-( $p$ - carboxyphenyl)-(naphtho- $1^{\prime} .2^{\prime}: 4.5$ triazolium)-chlorid(VI) ${ }^{6}$. Soweit die in Meerwasser $\left(p_{\mathrm{H}} 8,2-8,4\right)$ gelösten Substanzen bei den

s F. H. Straus, N. D. Cheronis u. E. Straus, Science [New York] 108, 113 [1948].

5 H.-J. B i e l i g, G. A. K a u s c h e u. H. H a a r d i c k, Z. Naturforschg. 4 b, im Druck [1949].

G E. L u d o l p hy, Diplomarbeit Univ. Heidelberg 1947.

7 D. Jerchel u. H. Fischer, Liebigs Ann. Chem., im Druck [1949]. 
angegebenen Konzentrationen von den Tieren zu den zugehörigen Formazanen bzw. Amino-azoverbindungen reduziert werden, erkennt man die Färbung frühestens nach 5 , spätestens nach 24 Stdn. Da die Farbstoffbildung z. Tl. auch durch Licht allein hervorgerufen wird, wurden die Lösungen während des ganzen Versuches dunkel gehalten.

In einer 0,01-proz. Lösung von I und in einer 0,2-proz. Lösung von III färben sich bei aus der Schale herauspräparierten, lebenden Pantoffel- analysiert. Bei Ephelota gemmipara R. Hertw. (Suctoria) färbt sich in I das ganze Tier mit Ausnahme des Stieles. Verschiedene nicht näher bestimmte Ciliaten ließen keine vitale Formazanbildung erkennen.

Unbefruchtete Eier des Seeigels Psammechinus miliaris $L$. zeigen unter der Einwirkung von 0,1 proz. I, weniger ausgeprägt und verzögert in $0,2-$ proz. V, im allgemeinen eine charakteristische Färbung der Keime unter Aussparung eines Poles. Von künstlich befruchteten Eiern desselben Tie-
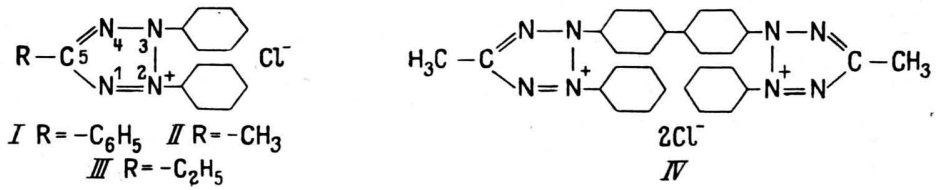

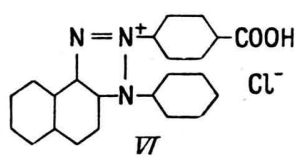

schnecken (Crepidula fornicata Lam.) das ventrale und dorsale Gebiet des Fußes, die Mantelrinne und die Niere zunehmend rot bzw. orangerot. Abgesonderter Schleim ist ebenfalls durch die Formazane angefärbt. Demgegenüber wird das Natriumsalz von VI bevorzugt von der Niere vital zum entsprechenden Azofarbstoff reduziert.

Veligerlarven von $C$. fornicata, die in 0,01-proz. I drei Tage lang beweglich bleiben, zeigen nach etwa 48 Stdn. im Gebiet der Mitteldarmdrüse leuchtend rote Färbung, während das übrige Tier keine Formazanbildung erkennen läßt. Später und weniger intensiv bildet sich an denselben Stellen rotes Formazan aus 0,2-proz. V.

Bei der Schnecke Tergipes Edwarsii (Opisthobranchia, Nudibranchiata) wird orangerotes Formazan von III (0,1-proz. Lösung) im Leben allein von einem Kranz Kleptokniden-führender Zellen gebildet, die sich im distalen Drittel der Kiemensäcke befinden.

Die Medusenknospen in den Gonotheken von Campanularia verticellata $L$. färben sich, abhängig vom Entwicklungszustand, die jüngeren intensiver als die älteren, in 0,1-proz. I rot, in 0,2-proz. III orangerot. Die Polypen bilden dabei kein Formazan.

Die Orte vitaler Reduktion bei der Garneele Crangon vulgaris F. in II, der Anthozoe Sagartia spez. (Gosse) in' I und III und der Muschel Petricola lithophaga Retz in I wurden noch nicht näher res, die sich, nach der Besamung in 0,05-proz. I übertragen, zu etwa $20 \%$ bis zum Zweizellenstadium und zu etwa $10 \%$ bis zum Vierzellenstadium entwickeln, weisen die unentwickelten Keime teils totale Anfärbung, teils elektive Formazanbildung in einer äquatorialen Zone auf. Die Rötung ist hier in vergleichbaren Zeiten geringer als bei den unbefruchteten Eiern. Bei den Zweizellstadien finden sich verschiedene Varianten elektiver Rotfärbung. Allgemein diffuse Formazanablagerung beobachtet man im Vierzellstadium und bei in die Lösung eingebrachten höheren Entwicklungsstadien bis zur Blastula.

In 0,05- bis 0,1-proz. I und in 0,1-proz. III hebt sich bei den ersten Furchungstadien der Muschel Teredo navalis $L$. die große D-Zelle durch stärkere Formazanbildung von den übrigen Keimzellen ab. Auch bei dem Sandröhrenwurm Sabellaria spinulosa Leuck. findet man unter der Einwirkung entsprechender Konzentrationen von I Unterschiede in der Lage der "Reduktionsorte“ zwischen unbefruchteten Eiern, befruchteten Eiern und frühen Furchungsstadien. Ihre Analyse wird indessen durch lokalisiertes, rosarotes Eigenpigment sehr erschwert.

Im Gegensatz zum Stiersperma ${ }^{3}$ bilden bei $P s$. microtuberculatus nur die Spermatozoen Formazan von I, nicht indessen der Spermaliquor.

Unabhängig von der Art nimmt die Giftigkeit der Tri- und Tetrazoliumsalze bei den hier unter- 
suchten Objekten etwa in der Reihenfolge I, IV, III, II, V, VI ab. Es entwickeln sich z. B. befruchtete Eier von Ps. miliaris in vergleichbaren Zeiten bis zur annähernd normalen Gastrula in 0,01proz. I, 0,05-proz. III und IV, 0,1-proz. II und 0,2proz. V und VI. Unbefruchtete Eier desselben Tieres, die 8 Stdn. lang in einer 0,1-proz. Lösung von II gehalten werden, erweisen sich anschließend in Meerwasser noch als befruchtbar, nicht dagegen solche, die der gleichen Einwirkungszeit von 0,1-proz. I ausgesetzt waren. Höhere als die angegebenen Konzentrationen der einzelnen Stoffe führen, je nach Objekt, in kürzerer oder längerer Zeit zu dessen Absterben. Tierische Objekte scheinen danach gegen die Tetrazoliumsalze empfindlicher zu sein als die bisher geprüften pflanzlichen Objekte und Bakterien. Eine postmortale Formazanbildung erfaßt den ganzen Körper des Tieres. An der Zool. Station in Neapel durchgeführte Versuche mit vor und nach der Einwirkung von I zentrifugierten Eiern von Ps. microtuberculatus legen nahe, daß an der Ausbildung der beobachteten, elektiven Anfärbungen Entmischungsvor gänge beteiligt sind.

Gegenüber den zum Studium der Oxydo-Reduktionsvorgänge bei Tieren bisher benutzten reversibel hydrier- und dehydrierbaren Farbstoffen (Methylenblau, Janusgrün, Brillantkresylblau u. a.) ${ }^{8}$ haben die oben verwendeten Tri- und Tetrazoliumsalze den Vorteil einer irreversiblen, farbigen „Leukostufe“, die in Wasser unlöslich und gegenüber Sauerstoff beständig ist. Von Nachteil ist bei den von uns bisher untersuchten derartigen Verbindungen ihre höhere Toxizität und die geringere Bildungsgeschwindigkeit der gefärbten Reduktionsstufen.

8 C. M. Ch i l d , Protoplasma 22, 377 [1935]; Roux' Arch. Entwicklungsmech. 136, 169 [1936]. - F. G. $\mathrm{F}$ i s c h e r u. H. H a r t w i g, Z. vergl. Physiol. 24, 1 [1936]. - E. R i e s, Naturwiss. 25, 241 [1937]. M. Gerseh u. E. Ries, Roux' Arch. Entwicklungsmech. 136, 169 [1937]. - H. P i e p h o, Biol. Zbl. 58, 90 [1938].

\title{
- Über die ungeordnete Bewegung von Bakterien
}

\author{
Von Hermann Engelhard und Thea Houtermans \\ Aus dem Hygienischén Institut der Universität Göttingen \\ (Z. Naturforschg. 4 b, 23-26 [1949]; eingegangen am 18. Oktober 1948)
}

Es wird die ungeordnete Bewegung von Bakterien untersucht und aus ihr die spezifische Molekülzahl ermittelt.

$\mathrm{D}$ ie Bewegung von Bakterien wird bei bakteriologischen Untersuchungen ständig beobachtet und ist vielfach beschrieben worden. Man unterscheidet die Bewegung von Bakterien ohne Bewegungsorganellen, die als Brownsche Bewegung seit langem angesehen wird und von K. Przi$\mathrm{bram}^{\mathbf{1}}$ an toten Bakterien gemessen wurde, sowie die von Bakterien mit Bewegungsorganellen, vorwiegend Geißeln, die sich selbständig bewegen. Eingehende Untersuchungen über die Bewegung der nicht selbständig beweglichen, meist unbeweglich genannten Bakterien sind nicht bekannt. Lediglich v. Ange $\mathrm{rer}^{2}$ hat die Senkungsgeschwindigkeit von Ruhrbazillen, also ihre Fallgeschwindigkeit, bestimmt. Die klassischen

1 K. Przibram, S.-B. Akad. Wiss. Wien, Abt. II a 121, 2340 [1912].

2 v. A n g e r e r, Arch. Hyg. Bakteriol. 88, 139 [1919].

3 J. Perrin, Die Atome. Theodor Steinkopff, Leipzig 1914.
Untersuchungen von $\mathrm{Per} r n^{3}$ über die Bewegung von Kolloiden ließen es daher reizvoll erscheinen, diese auch auf Bakterien auszudehnen.

Als Versuchsobjekt wurden Luftkokken wegen ihrer nahezu kugelförmigen Gestalt und etwa gleichen Größe gewählt, und an ihnen der Radius nach den für Kolloide geltenden Gesetzen auf zwei verschiedene Arten errechnet:

A. Aus der barometrischen Höhenverteilung einer sich im Gleichgewicht befindlichen Aufschwemmung.

B. Aus dem mittleren Verschiebungsquadrat. Ein Vergleich dieser Werte mit dem auf direktem Wege im Elektronenmikroskop gemessenen Radius ergab eine im Rahmen der Meßgenauigkeif befriedigende Übereinstimmung. Es ergibt sich danach eindeutig, daß dieser speziellen Art von unbeweglichen Bakterien, den Luftkokken, keinerlei Eigenbewegung zukommt. Analog zu der Bestimmung der spezifischen Molekülzahl nach Per- 